\title{
SCIENTIFIC REPORTS

\section{Neurometabolic Remodeling in Chronic Hiv Infection: a Five-Year Follow-up Multi-Voxel Mrs Study}

\author{
Jasmina Boban $\mathbb{1 D}^{1,2^{*}}$, Majda M. Thurnher ${ }^{3}$, Snezana Brkic ${ }^{1,4}$, Dajana Lendak ${ }^{1,3}$, \\ Vojislava Bugarski Ignjatovic ${ }^{1,5}$, Aleksandar Todorovic ${ }^{1,2}$ \& Dusko Kozic ${ }^{1,2}$
}

There is a lack of data about the long-term follow-up changes in neurometabolic profile and neuropsychological performance of HIV-positive subjects under continuous antiretroviral therapy (CART). The aim of the study was to assess changes in neurometabolic profile in chronically-infected, HIV-positive subjects during a five-year follow-up period, using multi-voxel proton magnetic resonance spectroscopy ( ${ }^{1} \mathrm{H}$-MRS). Nineteen neurologically asymptomatic, aviremic, HIV-positive subjects, underwent multi-voxel 2D MRS on a 3T MR unit and synchronous neurocognitive assessment in a five-year follow-up period. Twelve voxels were placed in prefrontal cortices, anterior and posterior cingulate gyrus, intraparietal sulci, and frontal centrum semiovale white matter, to identify peaks of $\mathrm{N}$-acetyl-aspartate (NAA), creatine (Cr), choline (Cho), and myoinositol (ml). Ratios of NAA/Cr, NAA/ $\mathrm{Cho}, \mathrm{NAA} / \mathrm{ml}, \mathrm{ml} / \mathrm{Cr}$, and $\mathrm{Cho} / \mathrm{Cr}$ were analyzed. Longitudinal differences in ratios and neurocognitive scores were tested with the Wilcoxon signed-rank-test. Statistical significance was set at $p \leq 0.004$ significant, and $0.05>p>0.004$ trending toward significance. A significant longitudinal increase in $\mathrm{NAA} / \mathrm{Cr}$ ratio was observed in $5 / 12$ voxels, while there was a trend toward significance in an additional three. The increase in Cho/ $\mathrm{Cr}$ reached statistical significance in one voxel. Changes in the $\mathrm{ml} / \mathrm{Cr}$ ratio demonstrated a significant increase in 4/12 voxels. A progressive increase in NAA/Cr, followed by better neurocognitive performance, may be an indicator of brain plasticity in the setting of chronic HIV-related neuronal injury. A progressive $\mathrm{ml} / \mathrm{Cr}$ increase could be partly explained by glial proliferation due to functional compartment remodeling and partly attributable to insufficient control of persistent neuroinflammation by CART.

Several longitudinal studies have shown changes in the biochemical profile of the brain in human immunodeficiency virus (HIV) infection, depending on the phase of infection and the introduction of combination antiretroviral therapy $(\mathrm{cART})^{1,2}$. Although no signs of neuronal injury can be detected on magnetic resonance imaging (MRI) and magnetic resonance spectroscopy (MRS) in the early phase of HIV transmission, there are already prominent signs of ongoing inflammation. This inflammation is reflected by elevated levels of myoinositol (mI), a marker of microglial proliferation, and choline (Cho), a marker of membrane metabolism and gliosis. Soon after the initiation of cART, in the absence of neuronal dysfunction, normalization of inflammation markers can occur in several weeks ${ }^{1}$. The initial increase in inflammation markers will decline after cART initiation, although they do not reach the level of healthy controls ${ }^{3}$. Further in the course of disease, a decline in N-acetyl aspartate (NAA), a neuronal marker, occurs and becomes the dominant finding in chronically infected HIV-patients ${ }^{4}$.

In the first MRS study performed in our institution on 110 subjects $(60 \mathrm{HIV}$-positive patients and 50 age- and gender-matched controls), the results clearly showed that HIV-associated neurodegeneration affects the whole volume of the brain. Furthermore, the results of that study, as well as other, smaller studies, confirmed ongoing inflammation under $\mathrm{CART}^{2}$. The neurodegenerative process associated with long-standing, well-controlled HIV infection results in progressive neurocognitive dysfunction ${ }^{5}$. The explanation for this process is thought

\footnotetext{
${ }^{1}$ University of Novi Sad, Faculty of Medicine Novi Sad, Hajduk Veljkova 3, 21000, Novi Sad, Serbia. ${ }^{2}$ Oncology Institute of Vojvodina, Center for Diagnostic Imaging, Put dr Goldmana 4, 21204, Sremska Kamenica, Serbia. ${ }^{3}$ Medical University of Vienna, Department fo Biomedical Imaging and Image-guided Therapy, Waehringer Guertel 18-20, 1090, Vienna, Austria. ${ }^{4}$ Clinical Center of Vojvodina, Clinic for Infectious Diseases, Hajduk Veljkova 1, 21000, Novi Sad, Serbia. ${ }^{5}$ Clinical Center of Vojvodina, Clinic for Neurology, Hajduk Veljkova 1, 21000, Novi Sad, Serbia. *email: jasmina.boban@mf.uns.ac.rs
} 


\begin{tabular}{|c|c|c|c|c|c|c|c|c|}
\hline \multirow[b]{2}{*}{ Variable } & \multirow[b]{2}{*}{$\mathbf{N}$} & \multirow[b]{2}{*}{ Mean } & \multirow[b]{2}{*}{ SD } & \multirow[b]{2}{*}{ Min } & \multirow[b]{2}{*}{$\operatorname{Max}$} & \multicolumn{3}{|c|}{ Percentiles } \\
\hline & & & & & & $25^{\text {th }}$ & $\begin{array}{l}\text { 50th } \\
\text { (Median) }\end{array}$ & $75^{\text {th }}$ \\
\hline Age (years) & 19 & 45.16 & 11.47 & 25 & 66 & 34.00 & 43.00 & 56.00 \\
\hline Education (years) & 19 & 12.21 & 3.39 & 4 & 16 & 10.00 & 12.00 & 16.00 \\
\hline $\begin{array}{l}\text { Nadir CD4 count } \\
\text { (cells/mL) }\end{array}$ & 19 & 267.32 & 208.62 & 11 & 694 & 105.00 & 200.00 & 460.00 \\
\hline $\mathrm{CPE}$ & 19 & 8.92 & 1.23 & 7 & 10 & 8.50 & 9.00 & 10.50 \\
\hline \multicolumn{9}{|c|}{ CD4 count (cells/mL) } \\
\hline 2011 & 19 & 558.58 & 296.280 & 210 & 1122 & 261.00 & 526.00 & 801.00 \\
\hline 2016 & 19 & 731.06 & 283.781 & 347 & 1195 & 450.00 & 754.00 & 984.00 \\
\hline \multicolumn{9}{|l|}{ cART (years) } \\
\hline 2011 & 19 & 5.65 & 2.25 & 1.5 & 11 & 2.25 & 5 & 8.75 \\
\hline 2016 & 19 & 10.65 & 2.25 & 6.5 & 16 & 7.25 & 10 & 13.75 \\
\hline
\end{tabular}

Table 1. Demographic and basic clinical data for the study participants at baseline and after five-year follow up.

to be due to chronic neuroinflammation and persistent immune activation, driven by an uneradicated pool of HIV-particles 6 .

In this longitudinal study, our aim was to assess longitudinal changes in the neurometabolic profile of neurologically asymptomatic HIV-positive individuals treated with cART during the follow-up period of five years, using multi-voxel proton magnetic resonance spectroscopy $\left({ }^{1} \mathrm{H}\right.$ MRS). In addition, a relationship between longitudinal changes in neurocognitive performance and MRS findings was investigated.

\section{Methods}

Participants. Baseline neuroimaging was performed in $31 \mathrm{HIV}$-positive patients, of whom 22 patients underwent follow-up imaging after five years. Three patients were excluded from the study based on the exclusion criteria. An overall total of 19 chronically infected HIV+ male patients on cART, mean age $45.16 \pm 11.47$, were included in this institutional review board-approved study (Ethical Committee of the Faculty of Medicine Novi Sad). Plasma viral load remained undetectable during the follow-up period in all patients. Participants underwent standard magnetic resonance imaging (MRI) and an MRS study on a 3 T MR unit (Trio Tim, Siemens, Erlangen, Germany), in the follow-up period of five years (e.g., baseline MRS studies were performed in 2011 and follow-up studies in 2016). Demographic and basic clinical data for both time points (including the nadir CD4 T-cell count, the current count of CD4+ T-cells, and cART duration) are summarized in Table 1.

Inclusion criteria for the follow-up study were: completion of two MR examinations and synchronous neuropsychological testing; chronic HIV infection at the baseline (over one year after transmission); stable on cART (over one year after initiation); no signs of manifest neurocognitive disorder at baseline; and a normal conventional MRI. Exclusion criteria were: detectable viral load over the follow-up period ( $>50$ copies $/ \mathrm{mL}$ ), current or past confounding neurological disease (opportunistic infection, multiple sclerosis, vascular and non-vascular dementia, neurodegenerative disease); brain tumors; traumatic brain injury; major psychiatric disorders; cardiovascular diseases (hypertension, chronic occlusive carotid disease, ischemic vascular dementia); active abuse of narcotic drugs (according to the Diagnostic and Statistical Manual of Mental Disorders); and hepatitis B or C coinfection at the baseline and over the follow-up period.

All selected patients were compliant with therapy, without any changes in the regimen of cART over the observed period of time. Three patients were excluded from follow-up imaging due to a change in the cART regimen (transient neuropsychiatric symptoms presented due to the introduction of efavirenz). A CONSORT study diagram showing patient selection in detail is shown in Fig. 1. For all subjects, the CNS penetrance efficacy score (CPE) was generated, with a mean value of $8.9 \pm 1.3$.

All subjects signed written, informed consent.

Neuroimaging protocol. Imaging was performed in 2011 and 2016, using an eight-channel head array coil. The MRI protocol consisted of a three-planar, multi-sequential study: sagittal T1-weighted spin echo (repetition time $440 \mathrm{~ms}$, time of echo $3.8 \mathrm{~ms}$, thickness of the slice $5 \mathrm{~mm}$ ); axial T2-weighted turbo spin echo ( $5150 \mathrm{~ms}, 105 \mathrm{~ms}, 5 \mathrm{~mm})$; FLAIR (FLuid Attenuation Inversion Recovery) $(8000 \mathrm{~ms}, 101 \mathrm{~ms}, 5 \mathrm{~mm}$ ); and coronal T2-weighted turbo spin echo (7150 ms, $111 \mathrm{~ms}, 5 \mathrm{~mm})$ MR tomograms.

Proton multi-voxel intermediate- $(1700 \mathrm{~ms}, 135 \mathrm{~ms})$ and short-echo-time (1700, $30 \mathrm{~ms}) \mathrm{MRS}$ data sets were acquired using a two-dimensional (2D) PRESS (Point RESolved Spectroscopy) technique, with automatic volume-selective shimming. Features of the spectroscopic slab were: volume of interest $80 \times 80 \times 10 \mathrm{~mm}$; thickness $10 \mathrm{~mm}$; and region of interest positioned in the supracallosal white and gray matter. The multi-voxel network consisted of 64 voxels, of which we chose 12 symmetrical voxels in both hemispheres:

1,2: the grey matter of the prefrontal cortex (PFC);

3,4 : the ventral part of the anterior cingulate gyrus (ACG);

5, 6: the frontal centrum semiovale white matter (FCSWM);

7, 8: the dorsal part of the ACG;

9, 10: the intraparietal sulcus (IPS); and

11, 12: the posterior cingulate gyrus (PCG) (Fig. 1). 


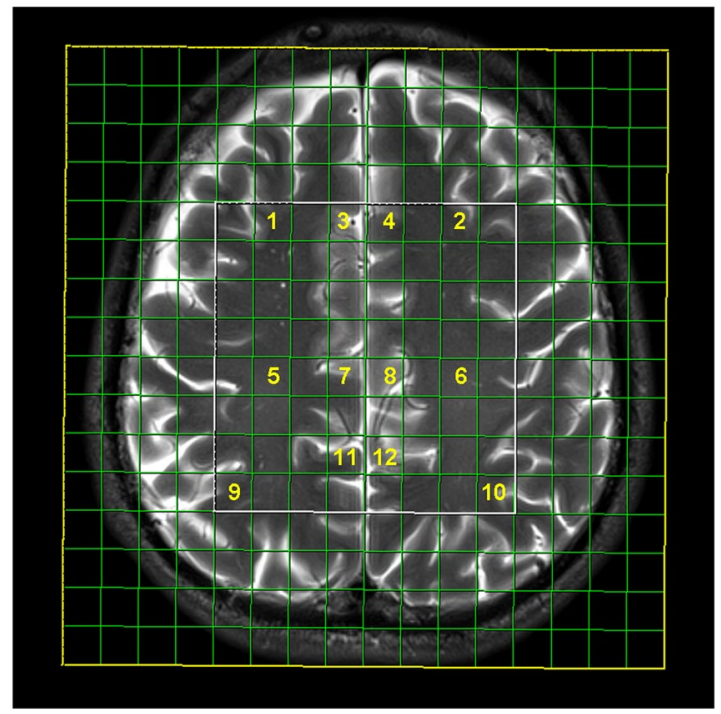

Figure 1. Multi-voxel network with analyzed voxel locations: prefrontal cortex in the right (1) and left (2) hemisphere; ventral anterior cingulate gyrus in the right (3) and left (4) hemisphere; dorsal anterior cingulate in the right (7) and left (8) hemisphere; centrum semiovale deep white matter in the right (5) and left (6) hemisphere; posterior cingulate gyrus in the right (11) and left (12) hemisphere; and intraparietal sulcus in the right (9) and left (10) hemisphere.

Post-processing was performed on the Leonardo workstation (Siemens, Erlangen, Germany) by two experienced neuroradiologists. The post-processing protocol consisted of baseline corrections, peak identification, and metabolite ratio calculations.

In each subject, two experienced neuroradiologists in consensus, selected the voxels that best covered the primary regions of interest (ROI), based on their location on the matching images (high-resolution). The investigators were blinded to the time of acquisition of the data. The mean of the peak area for observed metabolites were computed form fitted spectral data.

The peaks identified were: NAA; Cho; $\mathrm{mI}$; and total creatine $(\mathrm{Cr})$, used as a reference marker. Ratios of NAA/ $\mathrm{Cr}$ and $\mathrm{Cho} / \mathrm{Cr}$ on intermediate-echo time and $\mathrm{mI} / \mathrm{Cr}$ on short-echo time were analyzed.

Neurocognitive assessment. Synchronous follow-up neurocognitive testing was performed at the same time points as MRS. All participants underwent a 90-minute neuropsychological test battery that assessed multiple neurocognitive functions typically found to be impaired in HIV-positive subjects. This battery consisted of: the Mini Mental Score Examination $\left(\mathrm{MMSE}^{7}\right.$; the Trial making test form A and B (TmtA, TmtB) ${ }^{8}$; the Rey auditory-verbal learning test (RAVLT) ${ }^{9}$; the Rey-Osterrieth complex figure test $(\mathrm{ROCF})^{10}$; the Verbal fluency test-phonemic and categorical fluency tests ${ }^{11}$; the Verbal and visuospatial memory $\operatorname{span}^{12}$; the Wisconsin Sorting Card test ${ }^{13}$; and the Beck depression inventory scale $\mathrm{II}^{14}$. The same experienced clinical psychologist conducted all the neuropsychological testing. The study was conducted in compliance with the Helsinki Convention principles, was ethically approved, and every subject signed a written consent to participate in the research.

Statistical analysis. Statistical analysis was performed using IBM SPSS software (version 21.0, Chicago, IL, USA). Descriptive statistics included mean values, standard deviation, median, minimum and maximum for continuous and median values, range (minimum-maximum), and interquartile range (IQR) for variables that did not follow a normal distribution.

Metabolite ratios of NAA/Cr, Cho/Cr, and mI/Cr that were obtained on the MRS scans in 2011 and in 2016 were compared using the Wilcoxon signed-rank test for repeated measurements. Due to the suspected instability of $\mathrm{Cr}$, we performed longitudinal analysis calculations of NAA/Cho and NAA/mI ratios, in order to exclude the influence of $\mathrm{Cr}$ on metabolite ratios. The results of follow-up neurocognitive assessments performed at the same time as MRS were compared using the same test. We used this non-parametric test used because of the small sample size. For the same reason, we used Bonferroni corrections, designating p-values equal to or below 0.004 as significant (12 voxel locations, $0.05 / 12=0.004)$, and $0.05>\mathrm{p}>0.004$ as indicative of a trend toward significance.

Ethical approval. All procedures performed in studies involving human participants were in accordance with the ethical standards of the institutional and/or national research committee and with the 1964 Helsinki declaration and its later amendments or comparable ethical standards.

Informed consent. Informed consent was obtained from all individual participants included in the study. 


\begin{tabular}{|c|c|c|c|c|c|c|c|c|c|c|}
\hline \multirow[b]{2}{*}{$\mathrm{NAA} / \mathrm{Cr}$} & \multirow[b]{2}{*}{$\mathbf{N}$} & \multirow[b]{2}{*}{ Mean } & \multirow[b]{2}{*}{ SD } & \multirow[b]{2}{*}{ Min } & \multirow[b]{2}{*}{ Max } & \multicolumn{3}{|c|}{ Percentiles } & \multirow[b]{2}{*}{$Z^{*}$} & \multirow[b]{2}{*}{$\mathbf{p}^{*}$} \\
\hline & & & & & & $25^{\text {th }}$ & \begin{tabular}{|l} 
50th \\
Median
\end{tabular} & $75^{\text {th }}$ & & \\
\hline \multicolumn{11}{|c|}{ Right prefrontal cortex } \\
\hline 2011 & 19 & 1.636 & 0.229 & 1.21 & 2.16 & 1.480 & 1.690 & 1.800 & \multirow{2}{*}{-3.059} & \multirow{2}{*}{0.002} \\
\hline 2016 & 19 & 2.017 & 0.330 & 1.54 & 2.69 & 1.750 & 2.010 & 2.230 & & \\
\hline \multicolumn{11}{|c|}{ Right ventral ACG } \\
\hline 2011 & 19 & 1.402 & 0.252 & 0.83 & 1.91 & 1.220 & 1.350 & 1.600 & \multirow{2}{*}{-3.622} & \multirow{2}{*}{$<0.001$} \\
\hline 2016 & 19 & 1.890 & 0.173 & 1.58 & 2.24 & 1.740 & 1.870 & 1.980 & & \\
\hline \multicolumn{11}{|c|}{ Left ventral ACG } \\
\hline 2011 & 19 & 2.235 & 0.422 & 1.63 & 3.20 & 1.900 & 2.200 & 2.520 & \multirow{2}{*}{-0.644} & \multirow{2}{*}{0.520} \\
\hline 2016 & 19 & 2.141 & 0.294 & 1.63 & 2.56 & 1.890 & 2.210 & 2.380 & & \\
\hline \multicolumn{11}{|c|}{ Left prefrontal cortex } \\
\hline 2011 & 19 & 1.587 & 0.169 & 1.29 & 1.93 & 1.430 & 1.580 & 1.690 & \multirow{2}{*}{-3.159} & \multirow{2}{*}{0.002} \\
\hline 2016 & 19 & 1.955 & 0.351 & 1.27 & 2.53 & 1.680 & 2.030 & 2.150 & & \\
\hline \multicolumn{11}{|c|}{ Right centrum semiovale WM } \\
\hline 2011 & 19 & 2.514 & 0.503 & 1.74 & 3.52 & 2.020 & 2.490 & 2.880 & \multirow{2}{*}{-0.762} & \multirow{2}{*}{0.446} \\
\hline 2016 & 19 & 2.392 & 0.539 & 1.62 & 3.53 & 1.990 & 2.150 & 2.790 & & \\
\hline \multicolumn{11}{|c|}{\begin{tabular}{|l} 
Right dorsal ACG \\
\end{tabular}} \\
\hline 2011 & 19 & 1.993 & 0.291 & 1.58 & 2.67 & 1.760 & 1.970 & 2.110 & -1503 & 0133 \\
\hline 2016 & 19 & 1.769 & 0.300 & 1.18 & 2.16 & 1.540 & 1.760 & 1.990 & -1.503 & 0.133 \\
\hline Left dorsa & & & & & & & & & & \\
\hline 2011 & 19 & 1.483 & 0.282 & 0.72 & 1.92 & 1.370 & 1.530 & 1.680 & -2354 & 0019 \\
\hline 2016 & 19 & 1.781 & 0.380 & 1.07 & 2.67 & 1.530 & 1.720 & 2.020 & -2.354 & 0.019 \\
\hline Left centr & sem & ovale W & & & & & & & & \\
\hline 2011 & 19 & 1.440 & 0.405 & 1.00 & 2.94 & 1.240 & 1.330 & 1.510 & -3341 & م \\
\hline 2016 & 19 & 2.176 & 0.407 & 1.59 & 3.08 & 1.840 & 2.100 & 2.460 & -3.341 & 0.001 \\
\hline Right intr & Irieta & sulcus & & & & & & & & \\
\hline 2011 & 19 & 2.057 & 0.350 & 1.62 & 2.89 & 1.780 & 1.980 & 2.200 & -2435 & \\
\hline 2016 & 19 & 2.376 & \begin{tabular}{|l|}
0.380 \\
\end{tabular} & 1.59 & 3.01 & 2.160 & 2.400 & 2.690 & -2.435 & 0.015 \\
\hline \begin{tabular}{|l|} 
Right PCC \\
\end{tabular} & & & & & & & & & & \\
\hline 2011 & 19 & 1.516 & 0.189 & 1.03 & 1.85 & 1.420 & 1.480 & 1.620 & -3824 & $<0,001$ \\
\hline 2016 & 19 & \begin{tabular}{|l|}
1.947 \\
\end{tabular} & 0.236 & 1.62 & 2.45 & 1.740 & 1.920 & 2.140 & -3.824 & $<0.001$ \\
\hline Left PCG & & & & & & & & & & \\
\hline 2011 & 19 & 1.998 & 0.352 & 1.39 & 2.78 & 1.790 & 1.960 & 2.170 & -0.806 & 0.420 \\
\hline 2016 & 19 & 1.908 & 0.212 & 1.39 & 2.17 & 1.740 & 1.980 & 2.080 & & \\
\hline Left intrap & ietal & ulcus & & & & & & & & \\
\hline 2011 & 19 & 1.902 & 0.316 & 1.47 & 2.85 & 1.670 & 1.910 & 2.010 & -2.214 & 0.027 \\
\hline 2016 & 19 & 2.163 & 0.329 & 1.65 & 2.93 & 1.940 & 2.030 & 2.420 & -2.214 & 0.027 \\
\hline
\end{tabular}

Table 2. Longitudinal changes in NAA/Cr levels in named locations in the brain obtained on long-echo time MRS ( $\mathrm{p} \leq 0.004$ significant, $0.05>\mathrm{p}>0.004$ trending toward significance). *Wilcoxon signed-rank test. ACGanterior cingulate gyrus, WM-white matter, PCG-posterior cingulate gyrus.

\section{Results}

NAA/Cr ratios. A significant rise in the NAA/Cr levels in 5/12 analyzed voxels was observed in the following locations (Table 2): in the PFC on the left and right; in the right ventral ACG; in the left FCSWM; and in the right PCG $(\mathrm{p}=0.002, \mathrm{p}=0.002, \mathrm{p}<0.001, \mathrm{p}=0.001$ and $\mathrm{p}<0.001$, respectively). In the left dorsal ACG, the right IPS, and the left IPS, the increase trended toward significance $(\mathrm{p}=0.019, \mathrm{p}=0.015$ and $\mathrm{p}=0.027$, respectively).

Cho/Cr ratios. A significant increase in the Cho/Cr levels was detected in only one voxel, the right dorsal ACG $(\mathrm{p}=0.001)$. However, in several additional voxels, we observed an increase in Cho/Cr levels that trended toward statistical significance in the left PFC $(\mathrm{p}=0.013)$, in the left FCSWM $(\mathrm{p}=0.005)$, and in the left $(\mathrm{p}=0.007)$ and right IPS $(\mathrm{p}=0.009)$ (Table 3$)$.

$\mathrm{ml} / \mathrm{Cr}$ ratios. Longitudinal changes in $\mathrm{mI} / \mathrm{Cr}$ were heterogeneous. We observed an increase in the $\mathrm{mI} / \mathrm{Cr}$ level in the left ventral ACG $(p=0.002)$, the right dorsal ACG $(p=0.001)$, the right IPS $(p<0.001)$, and the right PCG $(p=0.001)$. In the left IPS ( $p=0.029)$, the increase trended toward significance. In the left dorsal ACG, and in the right and left FCSWM, we confirmed a decline in $\mathrm{mI} / \mathrm{Cr}(\mathrm{p}=0.033, \mathrm{p}=0.008$ and $\mathrm{p}=0.017$, respectively), but this only trended toward significance (Table 4 ). 


\begin{tabular}{|c|c|c|c|c|c|c|c|c|c|c|}
\hline \multirow[b]{2}{*}{ Cho/Cr } & \multirow[b]{2}{*}{$\mathbf{N}$} & \multirow[b]{2}{*}{ Mean } & \multirow[b]{2}{*}{ SD } & \multirow[b]{2}{*}{ Min } & \multirow[b]{2}{*}{ Max } & \multicolumn{3}{|c|}{ Percentiles } & \multirow[b]{2}{*}{$\mathbf{Z}^{*}$} & \multirow[b]{2}{*}{$\mathrm{p}^{*}$} \\
\hline & & & & & & 25th & $\begin{array}{l}50^{\text {th }} \\
\text { Median }\end{array}$ & $75^{\text {th }}$ & & \\
\hline \multicolumn{11}{|c|}{ Right prefrontal cortex } \\
\hline 2011 & 19 & 1.038 & 0.264 & 0.64 & 1.61 & 0.840 & 1.010 & 1.170 & \multirow{2}{*}{-0.698} & \multirow{2}{*}{0.485} \\
\hline 2016 & 19 & 0.991 & 0.127 & 0.76 & 1.31 & 0.890 & 0.990 & 1.070 & & \\
\hline \multicolumn{11}{|c|}{ Right ventral ACG } \\
\hline 2011 & 19 & 1.170 & 0.233 & 0.92 & 1.70 & 1.050 & 1.080 & 1.200 & \multirow{2}{*}{-1.787} & \multirow{2}{*}{0.074} \\
\hline 2016 & 19 & 1.050 & 0.128 & 0.75 & 1.34 & 0.980 & 1.050 & 1.120 & & \\
\hline \multicolumn{11}{|c|}{ Left ventral frontal ACG } \\
\hline 2011 & 19 & 1.038 & 0.174 & 0.79 & 1.49 & 0.890 & 1.040 & 1.160 & \multirow{2}{*}{-1.209} & \multirow{2}{*}{0.227} \\
\hline 2016 & 19 & 1.167 & 0.237 & 0.89 & 1.71 & 1.020 & 1.060 & 1.320 & & \\
\hline \multicolumn{11}{|c|}{ Left prefrontal cortex } \\
\hline 2011 & 19 & 0.954 & 0.0140 & 0.71 & 1.21 & 0.840 & 0.980 & 1.070 & \multirow{2}{*}{-2.476} & \multirow{2}{*}{0.013} \\
\hline 2016 & 19 & 1.063 & 0.131 & 0.84 & 1.41 & 0.990 & 1.050 & 1.090 & & \\
\hline \multicolumn{11}{|c|}{ Right centrum semiovale WM } \\
\hline 2011 & 19 & 0.860 & 0.187 & 0.60 & 1.27 & 0.680 & 0.870 & 1.030 & \multirow{2}{*}{-2.838} & \multirow{2}{*}{0.005} \\
\hline 2016 & 19 & 1.049 & 0.197 & 0.69 & 1.40 & 0.920 & 1.030 & 1.180 & & \\
\hline \multicolumn{11}{|c|}{\begin{tabular}{|l|} 
Right dorsal ACG \\
\end{tabular}} \\
\hline 2011 & 19 & 0.844 & 0.160 & 0.63 & 1.20 & 0.720 & 0.800 & 0.970 & -3200 & 0001 \\
\hline 2016 & 19 & 1.074 & 0.117 & 0.75 & 1.25 & 0.990 & 1.100 & 1.150 & -3.200 & 0.001 \\
\hline Left dor & al A & & & & & & & & & \\
\hline 2011 & 19 & 1.065 & 0.146 & 0.87 & 1.39 & 0.940 & 1.050 & 1.160 & & \\
\hline 2016 & 19 & 1.092 & 0.075 & 0.93 & 1.22 & 1.030 & 1.100 & 1.150 & -1.147 & 0.251 \\
\hline Left cen & rum & emioval & WM & & & & & & & \\
\hline 2011 & 19 & 1.209 & 0.212 & 0.90 & 1.66 & 1.040 & 1.160 & 1.340 & -1610 & \\
\hline 2016 & 19 & 1.088 & 0.131 & 0.85 & 1.28 & 1.010 & 1.120 & \begin{tabular}{|l|l|}
1.190 \\
\end{tabular} & -1.610 & 0.107 \\
\hline Right in & trapa & ietal sul & & & & & & & & \\
\hline 2011 & 19 & 0.901 & 0.122 & 0.68 & \begin{tabular}{|l|}
1.21 \\
\end{tabular} & \begin{tabular}{|l|}
0.810 \\
\end{tabular} & \begin{tabular}{|l|}
0.890 \\
\end{tabular} & \begin{tabular}{|l|}
0.990 \\
\end{tabular} & -2617 & \\
\hline 2016 & 19 & 1.052 & \begin{tabular}{|l|}
0.125 \\
\end{tabular} & \begin{tabular}{|l|}
0.89 \\
\end{tabular} & 1.35 & \begin{tabular}{|l|l|}
0.960 \\
\end{tabular} & \begin{tabular}{|l|}
1.030 \\
\end{tabular} & \begin{tabular}{|l|l|}
1.170 \\
\end{tabular} & -2.617 & 0.009 \\
\hline Right $P$ & & & & & & & & & & \\
\hline 2011 & 19 & 1.023 & 0.145 & \begin{tabular}{|l|}
0.71 \\
\end{tabular} & 1.31 & \begin{tabular}{|l|}
0.940 \\
\end{tabular} & 1.030 & 1.080 & & \\
\hline 2016 & 19 & 1.014 & 0.226 & \begin{tabular}{|l|l|}
0.67 \\
\end{tabular} & 1.54 & \begin{tabular}{|l|l|}
0.850 \\
\end{tabular} & \begin{tabular}{|l|}
0.960 \\
\end{tabular} & \begin{tabular}{|l|l|}
1.130 \\
\end{tabular} & -0.262 & 0.794 \\
\hline Left PC & & & & & & & & & & \\
\hline 2011 & 19 & \begin{tabular}{|l|l|}
0.793 \\
\end{tabular} & 0.218 & 0.49 & \begin{tabular}{|l|}
1.27 \\
\end{tabular} & \begin{tabular}{|l|}
0.620 \\
\end{tabular} & \begin{tabular}{|l|l|}
0.790 \\
\end{tabular} & \begin{tabular}{|l|}
0.910 \\
\end{tabular} & & \\
\hline 2016 & 19 & 0.911 & \begin{tabular}{|l|}
0.143 \\
\end{tabular} & \begin{tabular}{|l|}
0.72 \\
\end{tabular} & $\mid 1.17$ & \begin{tabular}{|l|l|}
0.790 \\
\end{tabular} & \begin{tabular}{|l|}
0.870 \\
\end{tabular} & \begin{tabular}{|l|}
1.050 \\
\end{tabular} & -1.590 & 0.112 \\
\hline Left int & apari & tal sulct & & & & & & & & \\
\hline 2011 & 19 & \begin{tabular}{|l|}
0.790 \\
\end{tabular} & \begin{tabular}{|l|l|}
0.159 \\
\end{tabular} & 0.52 & 1.11 & \begin{tabular}{|l|}
0.670 \\
\end{tabular} & \begin{tabular}{|l|}
0.800 \\
\end{tabular} & \begin{tabular}{|l|}
0.920 \\
\end{tabular} & -2677 & 0007 \\
\hline 2016 & 19 & 1.003 & 0.258 & 0.64 & 1.80 & \begin{tabular}{|l|l|}
0.850 \\
\end{tabular} & \begin{tabular}{|l|l|}
0.960 \\
\end{tabular} & 1.150 & $-2.6 / 7$ & $0.00 \%$ \\
\hline
\end{tabular}

Table 3. Longitudinal changes in Cho/Cr levels in named locations in the brain obtained on long-echo time MRS ( $\mathrm{p} \leq 0.004$ significant, $0.05>\mathrm{p}>0.004$ trending toward significance). *Wilcoxon signed-rank test. ACGanterior cingulate gyrus, WM-white matter, $\mathrm{PCG}$ - posterior cingulate gyrus.

NAA/Cho ratios. In the right frontal ACG $(p<0.001)$, the left PFC $(p<0.001)$, the left FCSWM $(p<0.001)$, the right IPS $(p=0.001)$, and the right PCG $(p=0.001)$, we observed a significant increase in the NAA/Cho ratio, while, in the right PFC and the left PCG, the increase trended toward significance $(p=0.011$ and $p=0.009$, respectively). However, in the right FCSWM and the dorsal ACG on the same side, a significant decline in this ratio was observed $(\mathrm{p}=0.001$ and $\mathrm{p}=0.001$, respectively), as well as a decrease in the left PCG $(\mathrm{p}=0.009)$ that trended toward significance.

NAA/ml ratios. In the right ventral ACG, the left FCSWM, the right IPS, and the right PCG $(\mathrm{p}=0.003$, $\mathrm{p}<0.001, \mathrm{p}=0.001$ and $\mathrm{p}<0.001$, respectively), a significant increase in NAA $/ \mathrm{mI}$ was observed. A decrease in the NAA $/ \mathrm{mI}$ ratio was significant in the right FCSWM $(\mathrm{p}=0.003)$ and in the right dorsal ACG that trended toward significance $(\mathrm{p}=0.009)$.

Neurocognitive assessment. In the five-year follow-up period, there was a trend toward a better performance for functions of delayed recall and recognition of verbal material (RAVLT A7, RAVLT recognition list A), for tests of visuospatial orientation abilities (ROCF), and for visuospatial span (WMS-R visual span: backward and forward span) (Table 5). The results of the other neurocognitive tests showed no significant longitudinal differences. 


\begin{tabular}{|c|c|c|c|c|c|c|c|c|c|c|}
\hline \multirow[b]{2}{*}{$\mathrm{mI} / \mathrm{Cr}$} & \multirow[b]{2}{*}{$\mathbf{N}$} & \multirow[b]{2}{*}{ Mean } & \multirow[b]{2}{*}{ SD } & \multirow[b]{2}{*}{ Min } & \multirow[b]{2}{*}{ Max } & \multicolumn{3}{|c|}{ Percentiles } & \multirow[b]{2}{*}{$Z^{*}$} & \multirow[b]{2}{*}{ p* } \\
\hline & & & & & & $25^{\text {th }}$ & $\begin{array}{l}\mathbf{5 0}^{\text {th }} \\
\text { Median }\end{array}$ & 75th & & \\
\hline \multicolumn{11}{|c|}{ Right prefrontal cortex } \\
\hline 2011 & 19 & 0.483 & 0.184 & 0.22 & 0.87 & 0.320 & 0.480 & 0.650 & \multirow{2}{*}{-1.699} & \multirow{2}{*}{0.089} \\
\hline 2016 & 19 & 0.708 & 0.448 & 0.32 & 1.91 & 0.440 & 0.560 & 0.750 & & \\
\hline \multicolumn{11}{|c|}{ Right ventral ACG } \\
\hline 2011 & 19 & 0.674 & 0.238 & 0.34 & 1.19 & 0.520 & 0.650 & 0.840 & \multirow{2}{*}{-1.894} & \multirow{2}{*}{0.058} \\
\hline 2016 & 19 & 0.553 & 0.151 & 0.28 & 0.90 & 0.430 & 0.560 & 0.650 & & \\
\hline \multicolumn{11}{|c|}{ Left ventral ACG } \\
\hline 2011 & 19 & 0.465 & 0.148 & 0.20 & 0.66 & 0.320 & 0.460 & 0.580 & \multirow{2}{*}{-3.081} & \multirow{2}{*}{0.002} \\
\hline 2016 & 19 & 0.601 & 0.109 & 0.32 & 0.77 & 0.570 & 0.620 & 0.660 & & \\
\hline \multicolumn{11}{|c|}{ Left prefrontal cortex } \\
\hline 2011 & 19 & 0.587 & 0.121 & 0.39 & 0.80 & 0.510 & 0.600 & 0.650 & -0.926 & \multirow{2}{*}{0.354} \\
\hline 2016 & 19 & 0.649 & 0.160 & 0.43 & 1.05 & 0.530 & 0.640 & 0.790 & & \\
\hline \multicolumn{11}{|c|}{ Right centrum semiovale WM } \\
\hline 2011 & 19 & 0.402 & 0.154 & 0.20 & 0.81 & 0.250 & 0.400 & 0.470 & \multirow{2}{*}{-2.657} & \multirow{2}{*}{0.008} \\
\hline 2016 & 19 & 0.556 & 0.160 & 0.23 & 0.83 & 0.420 & 0.560 & 0.680 & & \\
\hline \multicolumn{11}{|c|}{ Right dorsal ACG } \\
\hline 2011 & 19 & 0.391 & 0.147 & 0.21 & 0.89 & 0.310 & 0.350 & 0.430 & \multirow{2}{*}{-3.220} & م \\
\hline 2016 & 19 & 0.614 & 0.122 & 0.30 & 0.78 & 0.550 & 0.590 & 0.720 & & 0.001 \\
\hline Left do & & & & & & & & & & \\
\hline 2011 & 19 & 0.725 & 0.218 & 0.30 & 1.09 & 0.540 & 0.750 & 0.850 & -2.134 & 0.033 \\
\hline 2016 & 19 & 0.598 & 0.131 & 0.35 & 0.81 & 0.470 & 0.590 & 0.720 & & \\
\hline Left cer & emio & vale $\mathrm{WM}$ & & & & & & & & \\
\hline 2011 & 19 & 0.712 & 0.180 & 0.31 & 1.09 & 0.610 & 0.710 & 0.830 & 2 & م017 \\
\hline 2016 & 19 & 0.575 & 0.209 & 0.15 & 1.20 & 0.460 & 0.570 & 0.620 & -2.392 & 0.017 \\
\hline Right i & rietal & ulcus & & & & & & & & \\
\hline 2011 & 19 & 0.444 & 0.143 & \begin{tabular}{|l|l}
0.19 \\
\end{tabular} & 0.83 & 0.360 & 0.420 & 0.500 & 12 & - \\
\hline 2016 & \begin{tabular}{|l|}
19 \\
\end{tabular} & 0.650 & 0.146 & 0.34 & 0.85 & 0.540 & 0.680 & 0.770 & -3.543 & $<0.001$ \\
\hline Right $\mathrm{P}$ & & & & & & & & & & \\
\hline 2011 & 19 & 0.382 & 0.105 & 0.16 & 0.59 & 0.320 & 0.360 & 0.450 & & م 0000 \\
\hline 2016 & \begin{tabular}{|l|}
19 \\
\end{tabular} & \begin{tabular}{|l|}
0.607 \\
\end{tabular} & 0.148 & 0.32 & 0.92 & 0.500 & 0.610 & 0.720 & 315 & 0.001 \\
\hline Left PC & & & & & & & & & & \\
\hline 2011 & 19 & \begin{tabular}{|l|l|}
0.415 \\
\end{tabular} & 0.101 & \begin{tabular}{|l}
0.27 \\
\end{tabular} & 0.61 & 0.350 & 0.390 & \begin{tabular}{|l|}
0.470 \\
\end{tabular} & & 0240 \\
\hline 2016 & \begin{tabular}{|l|}
19 \\
\end{tabular} & 0.418 & 0.103 & 0.22 & 0.67 & 0.370 & 0.400 & 0.420 & 0.095 & 0.240 \\
\hline Left int & etal su & Icus & & & & & & & & \\
\hline 2011 & 19 & 0.395 & 0.165 & \begin{tabular}{|l|l}
0.07 \\
\end{tabular} & 0.66 & 0.230 & 0.390 & 0.550 & & \\
\hline 2016 & 19 & 0.510 & 0.186 & 0.20 & 0.90 & 0.380 & 0.480 & 0.630 & -2.179 & 0.029 \\
\hline
\end{tabular}

Table 4. Longitudinal changes in $\mathrm{mI} / \mathrm{Cr}$ levels in named locations in the brain obtained on short-echo time MRS ( $\mathrm{p} \leq 0.004$ significant, $0.05>\mathrm{p}>0.004$ trending toward significance). *Wilcoxon signed-rank test. ACGanterior cingulate gyrus, WM-white matter, $\mathrm{PCG}$ - posterior cingulate gyrus.

\section{Discussion}

Short-term longitudinal changes on MRS in the HIV-infected brain are well documented, and show a reduction of immune activation and inflammation in the brain parenchyma after the introduction of cART ${ }^{1}$. Despite the clear signs of neuroinflammation in the early phase of HIV infection, no signs of a neurodegenerative process have been observed ${ }^{1-3}$. Prior to the cART era, Chong et al. were the first to report a progressive reduction of the NAA level in HIV-positive subjects in a short follow-up period (three to eight months) ${ }^{15}$. Furthermore, based on later longitudinal studies, long-term control of both processes, neuroinflammation and consequent neurodegeneration, seemed to be suboptimal despite cART and good peripheral viral suppression ${ }^{16}$.

To the best of our knowledge, this is the first five-year follow-up longitudinal MR spectroscopic study on chronic, neurologically asymptomatic, aviremic HIV-positive patients, stable on cART (>one year at the baseline), with a normal MRI. According to the published data on neuropathogenesis of HIV infection and the suboptimal efficacy of cART in the brain, we expected to find signs of further, steady HIV-associated neuronal damage despite the reduction of an ongoing inflammatory process in patients with undetectable plasma viremia ${ }^{1,3,4}$.

Surprisingly, the results showed the expected, ongoing, low-level inflammation (reflected by a mI/Cr increase), and a clear long-term increase in NAA/Cr, suggesting the possibility of partial functional brain remodeling under cART. 


\begin{tabular}{|c|c|c|c|c|c|c|c|c|c|c|}
\hline \multirow[b]{2}{*}{ Test } & \multirow[b]{2}{*}{$\mathbf{N}$} & \multirow[b]{2}{*}{ Mean } & \multirow[b]{2}{*}{ SD } & \multirow[b]{2}{*}{ Min } & \multirow[b]{2}{*}{ Max } & \multicolumn{3}{|c|}{ Percentiles } & \multirow[b]{2}{*}{$Z^{*}$} & \multirow[b]{2}{*}{$\mathrm{p}^{*}$} \\
\hline & & & & & & 25th & \begin{tabular}{|l} 
50th \\
Median
\end{tabular} & $75^{\text {th }}$ & & \\
\hline \multicolumn{11}{|c|}{ MMSE } \\
\hline 2011 & 19 & 27.36 & 2.014 & 24 & 30 & 25.00 & 28.00 & 29.00 & \multirow{2}{*}{-1.179} & \multirow{2}{*}{0.238} \\
\hline 2016 & 19 & 28.36 & 2.335 & 24 & 30 & 27.00 & 29.00 & 30.00 & & \\
\hline \multicolumn{11}{|c|}{ TmtA } \\
\hline 2011 & 19 & 37.27 & 14.51 & 15 & 64 & 25.00 & 35.00 & 52.00 & \multirow{2}{*}{-0.460} & \multirow{2}{*}{0.654} \\
\hline 2016 & 19 & 36.09 & 16.85 & 17 & 70 & 23.00 & 33.00 & 46.00 & & \\
\hline TmtB & & & & & & & & & & \\
\hline 2011 & 19 & 54.18 & 26.17 & 22 & 113 & 36.00 & 42.00 & 73.00 & -0.059 & 0.953 \\
\hline 2016 & 19 & 54.45 & 26.61 & 20 & 98 & 34.00 & 53.00 & 87.00 & -0.059 & 0.950 \\
\hline RAVI & T A1 & & & & & & & & & \\
\hline 2011 & 19 & 49.00 & 7.823 & 38 & 59 & 42.00 & 47.00 & 58.00 & 15 & 0130 \\
\hline 2016 & 19 & 44.64 & 9.811 & 32 & 62 & 34.00 & 46.00 & 50.00 & -1.510 & 0.150 \\
\hline RAVI & $\Gamma$ list & & & & & & & & & \\
\hline 2011 & 19 & 10.00 & 3.493 & 5 & 15 & 8.00 & 9.00 & 14.00 & -1487 & 0137 \\
\hline 2016 & 19 & 8.36 & 3.472 & 2 & 15 & 6.00 & 9.00 & 10.00 & $-1.48 \%$ & $0.13 \%$ \\
\hline RAVI & $T$ list & & & & & & & & & \\
\hline 2011 & 19 & 7.45 & 3.012 & 3 & 14 & 5.00 & 8.00 & 9.00 & -2661 & 0008 \\
\hline 2016 & 19 & 10.09 & 2.737 & 7 & 15 & 7.00 & 10.00 & 12.00 & -2.001 & 0.008 \\
\hline RAVI & T rec & gnition & & & & & & & & \\
\hline 2011 & 19 & 10.91 & 2.212 & 6 & 14 & 10.00 & 11.00 & 13.00 & -2553 & 0011 \\
\hline 2016 & 19 & 13.64 & 2.063 & 9 & 15 & 12.00 & 15.00 & 15.00 & -2.353 & 0.011 \\
\hline Verba & flue & cy $S$ & & & & & & & & \\
\hline 2011 & 19 & 11.80 & 4.76 & 5 & 20 & 8.50 & 11.00 & 14.50 & -0298 & 0765 \\
\hline 2016 & 19 & 10.91 & 4.66 & 4 & 18 & 8.00 & \begin{tabular}{|l|l}
10.00 \\
\end{tabular} & \begin{tabular}{|l|l}
16.00 \\
\end{tabular} & -0.298 & 0.165 \\
\hline Verba & flue & cy $K$ & & & & & & & & \\
\hline 2011 & 19 & \begin{tabular}{|l|}
12.30 \\
\end{tabular} & 4.06 & 4 & 18 & 10.5 & 12.00 & 15.5 & -0358 & 0720 \\
\hline 2016 & 19 & \begin{tabular}{|l|l}
11.45 \\
\end{tabular} & \begin{tabular}{|l|l|}
4.72 \\
\end{tabular} & 6 & 20 & 7.00 & 11.00 & \begin{tabular}{|l|l}
15.00 \\
\end{tabular} & -0.358 & 0.720 \\
\hline Verba & flue & cy L & & & & & & & & \\
\hline 2011 & 19 & \begin{tabular}{|l|l|}
10.80 \\
\end{tabular} & 3.53 & 6 & 18 & 7.75 & 10.5 & 12.5 & -1542 & 0123 \\
\hline 2016 & 19 & \begin{tabular}{|l|}
8.82 \\
\end{tabular} & \begin{tabular}{|l|}
4.81 \\
\end{tabular} & 3 & 21 & 6.00 & \begin{tabular}{|l}
7.00 \\
\end{tabular} & 11.00 & -1.542 & 0.123 \\
\hline Verba & flue & cy anim & & & & & & & & \\
\hline 2011 & 19 & 22.20 & 6.29 & 16 & 34 & 17.75 & 19 & 29 & -1368 & 0171 \\
\hline 2016 & 19 & 20.36 & \begin{tabular}{|l|}
5.85 \\
\end{tabular} & 14 & 31 & \begin{tabular}{|l|l}
14.00 \\
\end{tabular} & \begin{tabular}{|l|l}
18.00 \\
\end{tabular} & 25.00 & -1.368 & 0.171 \\
\hline WCS & -cat & & & & & & & & & \\
\hline 2011 & 19 & 5.45 & 1.29 & 2 & 6 & 3.00 & 6.00 & 6.00 & 0756 & 0450 \\
\hline 2016 & 19 & \begin{tabular}{|l|l}
5.00 \\
\end{tabular} & \begin{tabular}{|l|l}
1.73 \\
\end{tabular} & 2 & 6 & 3.00 & \begin{tabular}{|l|l}
6.00 \\
\end{tabular} & 6.00 & -0.756 & 0.450 \\
\hline WCS & nos & & & & & & & & & \\
\hline 2011 & 19 & 1.18 & 2.09 & 0 & 7 & \begin{tabular}{|l|l} 
\\
\end{tabular} & 0.00 & 2.00 & 1280 & 0197 \\
\hline 2016 & 19 & \begin{tabular}{|l|}
0.36 \\
\end{tabular} & \begin{tabular}{|l|l|}
0.67 \\
\end{tabular} & 0 & 2 & \begin{tabular}{|l|l|}
0.00 \\
\end{tabular} & \begin{tabular}{|l|l|}
0.00 \\
\end{tabular} & \begin{tabular}{|l|l}
1.00 \\
\end{tabular} & -1.289 & 0.197 \\
\hline WCS & -per & & & & & & & & & \\
\hline 2011 & 19 & 12.55 & \begin{tabular}{|l|l}
10.32 \\
\end{tabular} & 3 & 38 & 5.00 & 9.00 & \begin{tabular}{|l|l}
19.00 \\
\end{tabular} & -0153 & 0878 \\
\hline 2016 & 19 & \begin{tabular}{|l|}
14.36 \\
\end{tabular} & \begin{tabular}{|l|}
12.35 \\
\end{tabular} & 3 & 42 & \begin{tabular}{|l}
5.00 \\
\end{tabular} & 10.00 & 26.00 & -0.153 & 0.878 \\
\hline Digit & pan & orward & & & & & & & & \\
\hline 2011 & 19 & \begin{tabular}{|l|}
6.91 \\
\end{tabular} & \begin{tabular}{|l|l}
0.539 \\
\end{tabular} & 6 & 8 & 7.00 & 7.00 & 7.00 & -1586 & 0113 \\
\hline 2016 & 19 & 6.18 & 1.250 & 4 & 8 & \begin{tabular}{|l|l|}
5.00 \\
\end{tabular} & \begin{tabular}{|l|l}
7.00 \\
\end{tabular} & $\begin{array}{l}7.00 \\
\end{array}$ & & \\
\hline Digit & pan & ackward & & & & & & & & \\
\hline \begin{tabular}{|l|l}
2011 \\
\end{tabular} & 19 & 5.55 & \begin{tabular}{|l|l}
1.440 \\
\end{tabular} & 4 & 8 & 4.00 & 5.00 & 7.00 & -0.997 & 0.319 \\
\hline 2016 & 19 & 5.00 & 1.342 & 3 & 7 & \begin{tabular}{|l|l|}
4.00 \\
\end{tabular} & \begin{tabular}{|l|l|}
5.00 \\
\end{tabular} & $\begin{array}{l}6.00 \\
\end{array}$ & & \\
\hline Visuo & pati & span fo & vard & & & & & & & \\
\hline 2011 & 19 & 5.36 & \begin{tabular}{|l|l|} 
\\
\end{tabular} & 4 & 7 & 5.00 & \begin{tabular}{|l|l|}
5.00 \\
\end{tabular} & 6.00 & -1925 & 0044 \\
\hline 2016 & 19 & \begin{tabular}{|l|}
6.18 \\
\end{tabular} & 1.250 & 4 & 8 & 5.00 & 6.00 & 7.00 & & 0.044 \\
\hline \begin{tabular}{|l} 
Visuo \\
\end{tabular} & pati & span ba & kward & & & & & & & \\
\hline 2011 & 19 & \begin{tabular}{|l|}
5.09 \\
\end{tabular} & \begin{tabular}{|l|l}
0.944 \\
\end{tabular} & 4 & 7 & 4.00 & 5.00 & 6.00 & -1978 & 0040 \\
\hline 2016 & 19 & 5.91 & 1.044 & 5 & 8 & 5.00 & 6.00 & 7.00 & -1.978 & 0.040 \\
\hline ROCI & copy & & & & & & & & & \\
\hline Con & inue & & & & & & & & & \\
\hline
\end{tabular}




\begin{tabular}{|c|c|c|c|c|c|c|c|c|c|c|}
\hline \multirow[b]{2}{*}{ Test } & \multirow[b]{2}{*}{$\mathbf{N}$} & \multirow[b]{2}{*}{ Mean } & \multirow[b]{2}{*}{ SD } & \multirow[b]{2}{*}{ Min } & \multirow[b]{2}{*}{ Max } & \multicolumn{3}{|c|}{ Percentiles } & \multirow[b]{2}{*}{$Z^{*}$} & \multirow[b]{2}{*}{$\mathbf{p}^{*}$} \\
\hline & & & & & & 25th & $\begin{array}{l}\text { 50th } \\
\text { Median }\end{array}$ & $75^{\text {th }}$ & & \\
\hline 2011 & 19 & 31.09 & 3.254 & 25.50 & 36.00 & 28.50 & 31.50 & 34.00 & \multirow{2}{*}{-2.456} & \multirow{2}{*}{0.014} \\
\hline 2016 & 19 & 33.86 & 2.491 & 28.00 & 36.00 & 34.00 & 34.00 & 36.00 & & \\
\hline \multicolumn{11}{|c|}{ BDI-II } \\
\hline 2011 & 19 & 8.45 & 6.039 & 2.00 & 21.00 & 3.00 & 8.00 & 11.00 & \multirow{2}{*}{-0.494} & \multirow{2}{*}{0.621} \\
\hline 2016 & 19 & 8.18 & 6.385 & 2.00 & 18.00 & 2.00 & 5.00 & 14.00 & & \\
\hline
\end{tabular}

Table 5. Longitudinal trends in specific neuropsychological tests obtained on the Wilcoxon signed-rank test (statistical significance set at $\mathrm{p}<0.05$ ). *Wilcoxon signed-rank test. MMSE-Mini Mental Score Examination, TmtA-Trial making test A (time in seconds), Tmt B-Trial making test B (time in seconds), RAVLT-Rey auditory-verbal learning test, verbal fluency S, L, K, animals-Serbian adaptation of verbal fluency FAS test, WCST-Wisconsin card sorting test, cat-categories, pers. err. -perseverative errors, ROCF-Rey-Osterrieth complex figure, BDI-II -Beck Depression Inventory II.

The development of HIV-associated neurodegeneration might be more complex than merely a pure consequence of a continuous and uncontrolled inflammatory process in the brain parenchyma. An increase in the NAA/Cr level was observed in almost all analyzed voxels in our study (Fig. 2), with no areas where a decrease was evident. The NAA/Cr level is used as a relevant marker of neuronal injury and dysfunction, with a marked decrease in all pathological processes in the brain, which imply a loss of viable neurons, as well as disorders in normal neuronal functioning ${ }^{17}$. The increase in NAA is rather an unusual finding; transiently, it occurs after acute brain injury, whereas a stable increase has been reported in moyamoya disease, amyotrophic lateral sclerosis, and Wernicke encephalopathy ${ }^{18,19}$. In all these conditions, the NAA increase suggested brain plasticity and partial functional recovery. Cysique et al. recently described longitudinal decrease in NAA in PCG and frontal white matter in HIV-positive persons with HAND (both mild neurocognitive disorder and asymptomatic neurocognitive disorder). However, stable intact subjects showed NAA levels closest to healthy controls, implying that HIV-positive persons with normal cognition are less affected by neurocognitive decline and decline in NAA levels in these two locations ${ }^{20}$. In our study sample, we observed not only that these levels did not decrease in 5-year period, but in some locations, they increased, which might speak in favor of plasticity in the light of concomitant neuropsychological testing.

A recent study by Sanford et al., conducted as a two-year follow-up MR volumetric study, showed no significant volume loss or reduction of cortical thickness in aviremic, stable, HIV-positive patients on cART, compared to HIV-negative controls ${ }^{21}$. The results and the patient selection criteria are in accordance with our findings.

The fact that no statistically significant longitudinal changes in $\mathrm{Cho} / \mathrm{Cr}$ were detected and statistical significance was reached in only one location (right dorsal ACG) in our study indicates good, long-term control of neuroinflammation under cART. In recent studies in chronic $\mathrm{HIV}$-infection, a progressive decline in $\mathrm{Cho} / \mathrm{Cr}$ was observed over time and explained as a probable reflection of tissue atrophy and a loss of brain parenchyma ${ }^{3}$. However, all these patients were symptomatic with severe neurocognitive disorders. This was not true for our patient population.

A transient increase in $\mathrm{mI} / \mathrm{Cr}$ was observed in acute (up to eight weeks after transmission) and primary HIV infection (up to six months after transmission) ${ }^{1}$. Sailasuta and Young showed a reduction in $\mathrm{mI} / \mathrm{Cr}$ soon after cART initiation; however, the levels of healthy controls were never reported ${ }^{1,3}$. Our finding of a dominant and stable increase in $\mathrm{mI} / \mathrm{Cr}$ levels indicates a continuous microglial proliferation, obviously insufficiently controlled by highly penetrative cART. In addition, a neurotoxic effect from long-standing cART might also be implied, concordant with a recent study by Winston et al..$^{22}$.

The increase in both $\mathrm{Cho} / \mathrm{Cr}$ and $\mathrm{mI} / \mathrm{Cr}$ ratios in our study may represent ongoing low-level inflammation and cell proliferation triggered by the presence of uneradicated HIV virus and its particles in the brain. This further suggests a suboptimal performance of cART in the brain, especially in the regions that have a significant number of microglial cells. This might explain the stable prevalence and incidence of milder forms of HIV-associated cognitive disorder in patients with cART.

Since the first studies on HIV-related brain injury, the diffuseness of the neurodegenerative process has been emphasized $^{23}$. A rather global increase in NAA/Cr was observed in our five-year follow-up study. Nevertheless, the increase in $\mathrm{mI} / \mathrm{Cr}$ was seen only in some locations (PFC, IPS, and FCSWM). This raises the question of whether certain brain regions are more susceptible to HIV injury due to complex pathways of HIV-associated neurodegeneration. Microglial cells are not equally distributed in the brain, with a variable density in the cerebral cortices, the brain stem, and the fiber bundles. Banati observed that persistent subtle microglial activity modulates neuronal functioning directly or indirectly, via astrocytic interaction. Microglial activation mobilizes functional compartments in which signaling molecules (neurokines, etc.) imply a more open cell-to-cell communication, not present in the healthy adult brain. These communications occur in neuronal pathways where this glial activation is under the influence of specific brain pathology, independent of disease etiology. This forms the framework for functional remodeling in the brain ${ }^{24}$.

During pathological process in the brain, the proliferation of microglia forms a network with regional differences that are not random, but functionally dependent ${ }^{25}$. Thus, microglial proliferation and membrane metabolism may be increased in those regions of the brain that constitute the brain circuits involved in attention and working memory, immediate and delayed recall, and visuospatial abilities, as a consequence of functional compartment remodeling in response to HIV-related chronic neuronal injury. The demonstrated increase in 


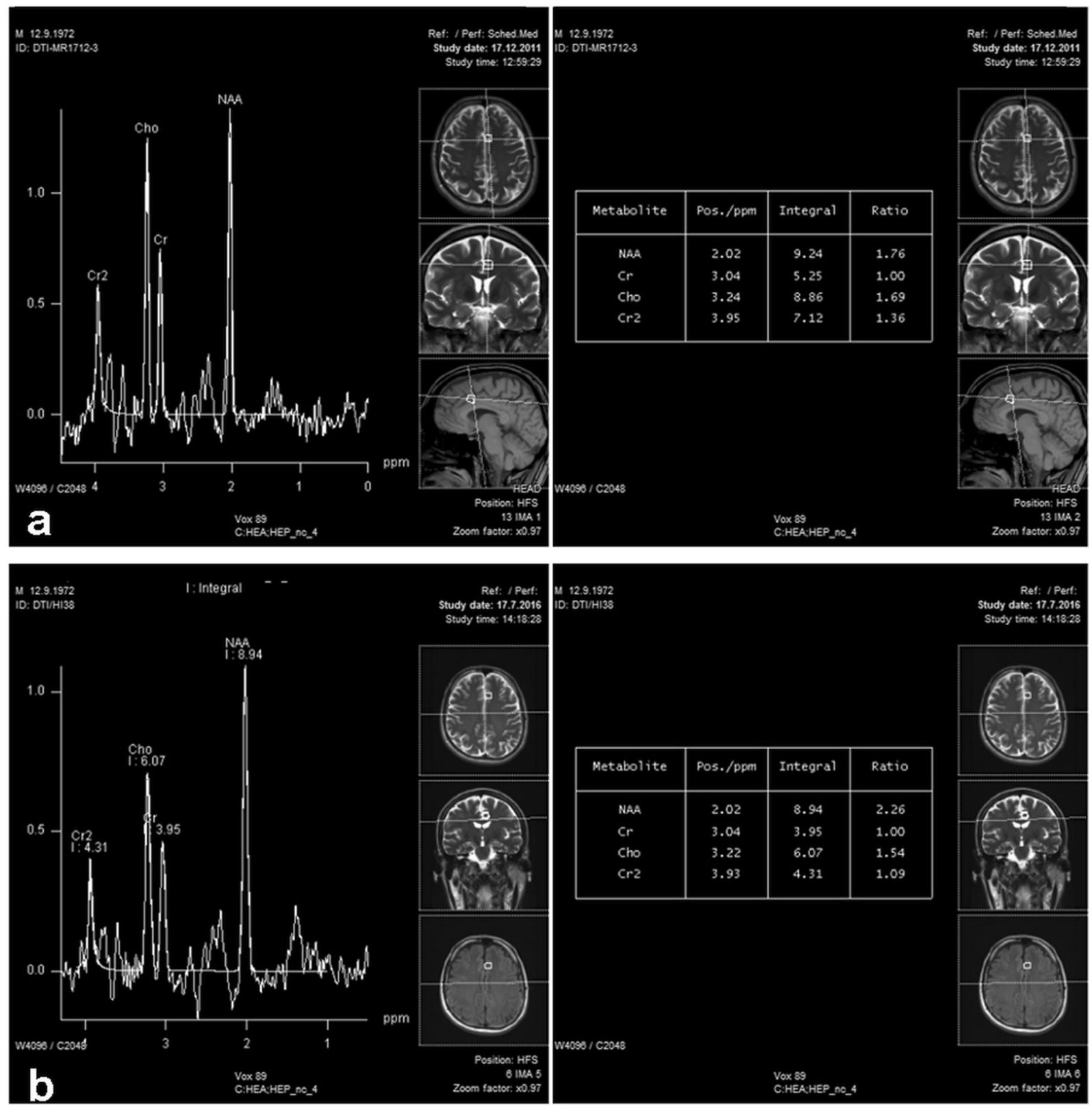

Figure 2. Follow-up long-echo time MR spectrum, performed in 2011 (a) and 2016 (b), with corresponding result tables, showing a progressive increase in NAA/Cr ratios.

neurobiochemical markers could represent proliferation of radial glial cells that lead neuronal migration to the site of injury. The dynamics of neurobiochemical changes observed in the locations analyzed in this study were supported by the results obtained in some neurocognitive tests. Namely, we observed better performance for the functions of immediate and delayed recall, regulated mostly by the dorsal ACG and parts of the PFC ${ }^{26,27}$. Dysfunction in connections to the ACG results in apathy, impulsivity, and disinhibition - behavioral changes that occur in neurodegenerative diseases. Finally, we observed better achievement in visual attention and working memory, regulated by many networks in the brain, mainly $\mathrm{PCG}^{28}$. It comes as no surprise that previous studies on HIV-positive subjects confirmed the most prominent reduction in NAA/Cr levels exactly in this region. In light of these findings, a question could be raised about the functional remodeling of the brain pathways involved in cognition, predominantly in attention/working memory, visuospatial abilities, and delayed recall and recognition of verbal material. Sanford et al., in a recently published two-year follow-up study, showed better interval performance on several neurocognitive tasks, especially TmtA, concordant with our study. The authors commented on the result as a probable beneficial effect of the on-time introduction of cART and long-standing stable aviremia ${ }^{21}$.

Limitations. The major limitation of the present study is the small number of participants, partially due to strict inclusion criteria. However, we performed a power analysis calculation for all three metabolite ratios. For NAA/Cr levels, the power analysis showed a satisfactory sample size (0.84-0.99), so that differences in NAA/Cr 
levels can be considered relevant despite a small sample size. The results of the power analysis for $\mathrm{Cho} / \mathrm{Cr}$ and $\mathrm{mI} / \mathrm{Cr}$ levels also showed a satisfactory sample size (0.98-0.99 and 0.86-0.99, respectively). Additional limitation is the partial volume effect of the cerebrospinal fluid, white matter or blood vessels) that can be present in some voxels that include mostly grey matter, such as voxels placed in cingulate gyrus. However, investigators that performed the MRS data processing controlled for this contamination and selected only spectra that best covered the ROIs with the grey matter. Finally, a significant issue might be the use of metabolite ratios instead of absolute values, given that the level of $\mathrm{Cr}$ is unstable in chronic HIV infected population, due to decreased uptake in the gut $^{29}$, or changes in the brain synthesis of $\mathrm{Cr}$.

\section{Conclusions}

In summary, an unexpected longitudinal increase in NAA/Cr in neurologically asymptomatic HIV-positive patients on cART, observed on multivoxel MRS, followed by better performance on neurocognitive tests with regard to attention/working memory, delayed recall, recognition and visuospatial abilities, suggests functional remodeling of brain circuits in HIV-related neurodegeneration. Increased microglial proliferation and membrane metabolism might be implied in cell-to-cell communication and functional remodeling as a response to chronic neuronal injury. However, the latter may also reflect persistent inflammation and immune activation, probably due to the suboptimal efficacy of cART in the brain. Regional differences in of $\mathrm{mI} / \mathrm{Cr}$ increases suggest that some brain regions are more prone to inflammation, either due to anatomical or neurophysiological features.

Received: 11 July 2019; Accepted: 2 December 2019;

Published online: 24 December 2019

\section{References}

1. Sailasuta, N. et al. Change in brain magnetic resonance spectroscopy after treatment during acute HIV infection. PloS One. 7(11), e49272 (2012).

2. Boban, J. et al. HIV-associated neurodegeneration and neuroimmunity: multivoxel MR spectroscopy study in drug-naive and treated patients. Eur Radiol. 27(10), 4218-4236 (2017).

3. Young, A. C. et al. Cerebral metabolite changes prior to and after antiretroviral therapy in primary HIV infection. Neurology. 83(18), 1592-1600 (2014).

4. Gongvatana, A. et al. Progressive cerebral injury in the setting of chronic HIV infection and antiretroviral therapy. J Neurovirol. 19, 209-218 (2013)

5. Clifford, D. B. \& Ances, B. M. HIV-associated neurocognitive disorder (HAND). Lancet Inf Dis. 13(11), 976-986 (2013).

6. Navia, B. A., Cho, E. S., Petito, C. K. \& Price, R. W. The AIDS dementia complex: II. Neuropathol. Ann Neurol. 19, $525-535$ (1986).

7. Folstein, M. F., Folstein, S. E. \& McHugh, P. R. Mini-mental state: a practical method for grading the cognitive state of patients for the clinician. J Psych Res. 12(3), 189-198 (1975).

8. Reiten, R. M. Validity of the Trial making test as an indicator of organic brain damage. Perceptual and motor skills. 8(3), 271-276 (1958).

9. Rey, A. L'examen dinique en psychologie. Paris: Presses Universitaires de France (1964).

10. Rey, A. L'examen psychologique dans les cas d'encephalopathie traumatique. Archives de psychologie (1941).

11. Pavlovic, D. M. Dijagnosticki testovi u neuropsihologiji, II izdanje. Beograd, GRAFOSBEOGRAD (2003).

12. Wechsler, D. \& Stone, C. P. Wechsler memory scale-revised. Psychological Corporation (1987).

13. Heaton, R. K. et al. Wisconsin Card Sorting test Manual: Revised and expanded edition. Odessa, Florida: Psycholocal Assessment Resoursec, Inc (1993).

14. Beck, A. T., Steer, R. A. \& Brown, G. K. Beck depression inventory- II. San Antonio. 78(2), 490-498 (1996).

15. Chong, W. K. et al. Localized cerebral proton MR spectroscopy in HIV infection and AIDS. AJNR AM J Neuroradiol. 15, 21-25 (1994).

16. Boban, J. et al. Proton Chemical Shift Imaging Study of the Combined Antiretroviral Therapy on Neurometabolic Parameters in Chronic HIV Infection. AJNR Am J Neuroradiol. 38(6), 1122-1129 (2017).

17. Barker, P. B. N-acetyl aspartate- a neuronal marker? Ann Neurol. 49(4), 423-424 (2001).

18. De Stefano, N., Matthews, P. M. \& Arnold, D. L. Reversible decreases in N-acetylaspartate after acute brain injury. Magn Reson Med. 34, 721-727 (1995).

19. Steen, R. G. \& Ogg, R. J. Abnormally high levels of brain N-acetyaspartate in children with sickle cell disease. AJNR Am J Neuroradiol. 26(3), 463-468 (2005).

20. Cysique, L. A. et al. Covertly active and progressing neurochemical abnormalities in suppressed HIV infection. Neurol Neuroimmunol Neuroinflamm. 5, e430 (2018).

21. Sanford, R., Fellows, L. K., Ances, B. M. \& Collins, L. Association of brain structure changes and cognitive function with combination antiretroviral therapy in HIV-positive individuals. JAMA Neurol. 75(1), 72-79 (2018).

22. Winston, A. et al. Differences in the direction of change of cerebral function parameters are evident over three years in HIV-infected individuals electively commencing initial cART. PLOS ONE. 10(2), e0118608 (2015).

23. Achim, C. L., Miners, D. K., Burrola, P. G., Martin, F. C. \& Wiley, C. A. In vivo model of HIV infection of the human brain. Dev Neurosci. 15(6), 423-432 (1993).

24. Banati, R. B. Brain plasticity and microglia: is transsynaptic glial activation in the thalamus after limb denervation linked to cortical plasticity and central sensitisation? J Physiol Paris. 96(3), 289-299 (2002).

25. Tay, T. L. et al. A new fate mapping system reveals context-dependent random or clonal expansion of microglia. Nat Neurosci. 20(6), 793-803 (2017).

26. Bonelli, R. M. \& Cummings, J. L. Frontal-subcortical circuitry and behaviour. Dialogues Clin Neurosci. 9(2), 141-151 (2007).

27. Stevens, F. L., Hurley, R. A. \& Taber, K. H. Anterior cingulate cortex: unique role in cognition and emotion. J Neuropsychiatry Clin Neurosci. 23(2), 121-125 (2011).

28. Leech, R. \& Sharp, D. J. The role of posterior cingulate cortex in cognition and disease. Brain. 137(Pt 1), 12-32 (2014).

29. Rae, C. D. \& Broer, S. Creatine as a booster for human brain infection. How might it work? Neurochem Int. 89, 249-259 (2015).

\section{Acknowledgements}

This study was supported by grant of Provincial Secretariat for Science and Technological Development of Autonomous Province of Vojvodina, number 114-451-2730/2016-01. 


\section{Author contributions}

All the authors have seen and approved the final version and have contributed significantly to the work with regard to conception and design (S.B. and J.B.), acquisition of the data (A.T. and V.B.I.), data analysis and interpretation (M.M.T., S.B., D.K., J.B., D.L. and V.B.I.), as well as drafting the article (J.B.) or revising it (M.M.T.). The requirements for the authorship according to your Journal have been met. All the authors agreed to the conditions noted on the Authorship Agreement forms. No writing assistance other than copy-editing was provided in the preparation of the manuscript.

\section{Competing interests}

The authors declare no competing interests.

\section{Additional information}

Correspondence and requests for materials should be addressed to J.B.

Reprints and permissions information is available at www.nature.com/reprints.

Publisher's note Springer Nature remains neutral with regard to jurisdictional claims in published maps and institutional affiliations.

(c) Open Access This article is licensed under a Creative Commons Attribution 4.0 International License, which permits use, sharing, adaptation, distribution and reproduction in any medium or format, as long as you give appropriate credit to the original author(s) and the source, provide a link to the Creative Commons license, and indicate if changes were made. The images or other third party material in this article are included in the article's Creative Commons license, unless indicated otherwise in a credit line to the material. If material is not included in the article's Creative Commons license and your intended use is not permitted by statutory regulation or exceeds the permitted use, you will need to obtain permission directly from the copyright holder. To view a copy of this license, visit http://creativecommons.org/licenses/by/4.0/.

(c) The Author(s) 2019 